\title{
COMPLICAÇÕES IMEDIATAS E TARDIAS APÓS CIRURGIA DE RESERVATÓRIO ILEAL NA POLIPOSE ADENOMATOSA FAMILIAR
}

\author{
Raquel Franco LEAL, Maria de Lourdes Setsuko AYRIZONO, Cláudio Saddy Rodrigues COY, \\ João José FAGUNDES e Juvenal Ricardo Navarro GÓES
}

\begin{abstract}
RESUMO - Racional - A retocolectomia total com confecção de reservatório ileal é cirurgia ideal para o tratamento do cólon e reto dos doentes com polipose adenomatosa familiar, no entanto pode estar associada a complicações no pós-operatório imediato e tardio. Objetivo - Estudar as complicações pós-operatórias da cirurgia do reservatório ileal na polipose adenomatosa familiar. Métodos - Estudo retrospectivo de 69 doentes com polipose adenomatosa familiar submetidos a cirurgia de reservatório ileal no período de 1984 a 2006, pelo Grupo de Coloproctologia da Faculdade de Ciências Médicas da Universidade Estadual de Campinas, SP. O seguimento médio pós-operatório foi de 82 (2-280) meses. Dados de interesse: ocorrência de complicações no pós-operatório. Resultados - A morbidade e mortalidade foram de $63,8 \%$ e $2,9 \%$, respectivamente. As complicações mais freqüentes foram obstrução intestinal (17,4\%), estenose da anastomose (15,9\%) e sepse pélvica (10,1\%). Outras complicações foram isquemia aguda do reservatório ileal (4,3\%), ileíte do reservatório ("pouchitis") $(2,9 \%)$ e fístulas relacionadas ao reservatório $(2,9 \%)$. Conclusões - A morbimortalidade foi semelhante à da literatura e aceitável para uma cirurgia complexa como é a do reservatório ileal, realizada em dois tempos operatórios. A obstrução intestinal foi a complicação mais freqüente. Entretanto, isquemia do reservatório, "pouchitis" e sepse pélvica constituíram importantes complicações relacionadas à perda do reservatório ileal.
\end{abstract}

DESCRITORES - Polipose adenomatosa do colo, cirurgia. Íleo, cirurgia. Reto, cirurgia. Bolsas do colo. Colectomia, complicações.

\section{INTRODUÇÃO}

A polipose adenomatosa familiar (PAF) é uma doença hereditária de caráter autossômico dominante, igualmente distribuída entre os sexos. Origina-se, principalmente, a partir da mutação na linhagem de células germinativas no gene da polipose adenomatosa coli (PAC), resultando em formação de centenas de pólipos colorretais. A principal característica clínica é a evolução para câncer colorretal em quase $100 \%$ dos casos, geralmente antes dos 40 anos de idade, se a cirurgia não for realizada. A incidência é de 1/7.000 nascimentos, no entanto, esta estimativa é incerta, uma vez que a doença não é óbvia ao nascimento e algumas vezes pode se manifestar na $4^{\mathrm{a}}$ ou $5^{\mathrm{a}}$ décadas de vida, nas formas atenuadas da doença $a^{(2,3,4,29)}$.

A retocolectomia total com confecção de reservatório ileal (RI) é a cirurgia de escolha para a PAF. Desde o seu advento, no final da década de $70^{(27,30,42)}$, o RI tem sido a reconstrução cirúrgica ideal nesses doentes, uma vez que possibilita a retirada de toda a mucosa do cólon e do reto com potencial de formação de pólipos, como também evita a ileostomia definitiva ${ }^{(18,21,42)}$.
Este procedimento está indicado nos doentes com PAF que possuem muitos pólipos retais, em geral mais do que 20 , ou presença de pólipos com displasia de alto grau no epitélio, condições predisponentes para desenvolvimento de câncer retal ${ }^{(17)}$.

Por ser cirurgia de maior complexidade, os doentes submetidos ao RI estão sujeitos a maior ocorrência de possíveis complicações tanto no pós-operatório imediato quanto tardio, quando comparados àqueles nos quais se faz apenas colectomia total e anastomose ileorretal. No entanto, de maneira geral, a retocolectomia total com RI apresenta aceitável morbidade e baixa mortalidade nos doentes portadores de $\operatorname{PAF}^{(9,18,21,24)}$.

Este estudo teve como objetivo demonstrar as complicações pós-operatórias da cirurgia do RI em doentes com PAF que possuíam acometimento colorretal, acompanhados no Ambulatório de Coloproctologia de hospital universitário e compará-las com dados da literatura.

\section{MÉTODOS}

Trata-se de estudo retrospectivo, descritivo, a partir da análise de prontuários. De janeiro de 1984 a dezembro de Grupo de Coloproctologia, Disciplina de Moléstias do Aparelho Digestório, Faculdade de Ciências Médicas, Universidade Estadual de Campinas, Campinas, SP.
Correspondência: Dra. Raquel Franco Leal - Rua Patativa, 170 - apt.24C - Condomínio Illhas do Pacífico - Bonfim - 13034-810 - Campinas, SP. E-mail: raquelleal@mpc.com.br 
2006, 69 doentes com PAF foram submetidos a retocolectomia total com RI, pelo Grupo de Coloproctologia do Hospital das Clínicas da Universidade Estadual de Campinas (UNICAMP), Campinas, SP.

Obtiveram-se dados gerais como sexo, idade, tempo de seguimento pós-operatório, técnica cirúrgica utilizada, além das complicações ocorridas no pós-operatório imediato (até 30 dias) e tardio.

Quarenta e um doentes $(59,4 \%)$ eram do sexo feminino, com média de idade, no momento da cirurgia, de 29,6 (13-58) anos. $\mathrm{O}$ achado anatomopatológico de adenocarcinoma colorretal in situ ocorreu em 10 doentes $(14,4 \%)$. Todos foram submetidos a mucosectomia e anastomose ileoanal manual por via endoanal, na linha pectínea, utilizando fio de poliglactina 3.0, sutura com pontos separados. Também foi realizada ileostomia de proteção ${ }^{(15)}$. Em $87 \%$ dos doentes, empregou-se a técnica de preservação da arcada do cólon direito ${ }^{(13)}$, como um suplemento à irrigação do RI.

Quanto ao tipo de reservatório utilizado, em 45 doentes $(65,2 \%)$ foi do tipo "J"(42), 15 (21,7\%) em "S" "(30) e 9 (13\%) em dupla câmara ${ }^{(12)}$. Os reservatórios em " $\mathrm{S}$ " e em dupla câmara foram confeccionados com sutura manual, pontos contínuos com fio de poliglactina 3.0 e os em "J", em sua maioria (35 casos $-77,8 \%$ ), foi realizada sutura com grampeador linear.

Os critérios de inclusão foram doentes com PAF submetidos a cirurgia de RI. Os doentes que perderam seguimento não foram excluídos e nestes foram analisadas as complicações até o momento da perda do acompanhamento em ambulatório. O tempo médio de pós-operatório foi de 82 (2-280) meses, sendo que sete doentes perderam seguimento após 3 anos de acompanhamento.

\section{RESULTADOS}

Doze doentes (17,4\%) evoluíram com obstrução intestinal que necessitou de laparotomia. A causa da obstrução foi aderência com acotovelamento de alças de delgado em nove casos, obstrução no local da ileostomia em outros dois e por tumor desmóide em outro. Este último, localizado no mesentério do delgado, foi considerado irressecável à laparotomia e a doença está controlada por meio do uso de antiinflamatório não-esteroidal e tamoxifeno. Não houve complicações relacionadas a estas reoperações (Figura 1).

Estenose cicatricial da anastomose ileoanal ocorreu em 11 casos (15,9\%), sendo necessária dilatação com resolução do quadro.

Sete doentes $(10,1 \%)$ evoluíram com sepse pélvica, necessitando de drenagem por via abdominal ou via pós-anal. Em dois casos foi necessária a retirada do RI da pelve e sua exteriorização na parede abdominal como ostomia (Figura 2).

Isquemia do RI ocorreu em três doentes $(4,3 \%)$. Um necessitou de laparotomia no $2^{\circ}$ dia de pós-operatório, com exteriorização do mesmo na parede abdominal em forma de ostomia. Outro apresentou isquemia crônica, evoluindo com estenose do reservatório. No terceiro doente ocorreu eliminação espontânea do RI após a necrose, no $30^{\circ}$ dia de pós-operatório. Não houve mortalidade e a cirurgia de re-reservatório ileal foi empregada em dois destes doentes após 35 meses, em média, da primeira cirurgia, com boa evolução imediata e tardia.

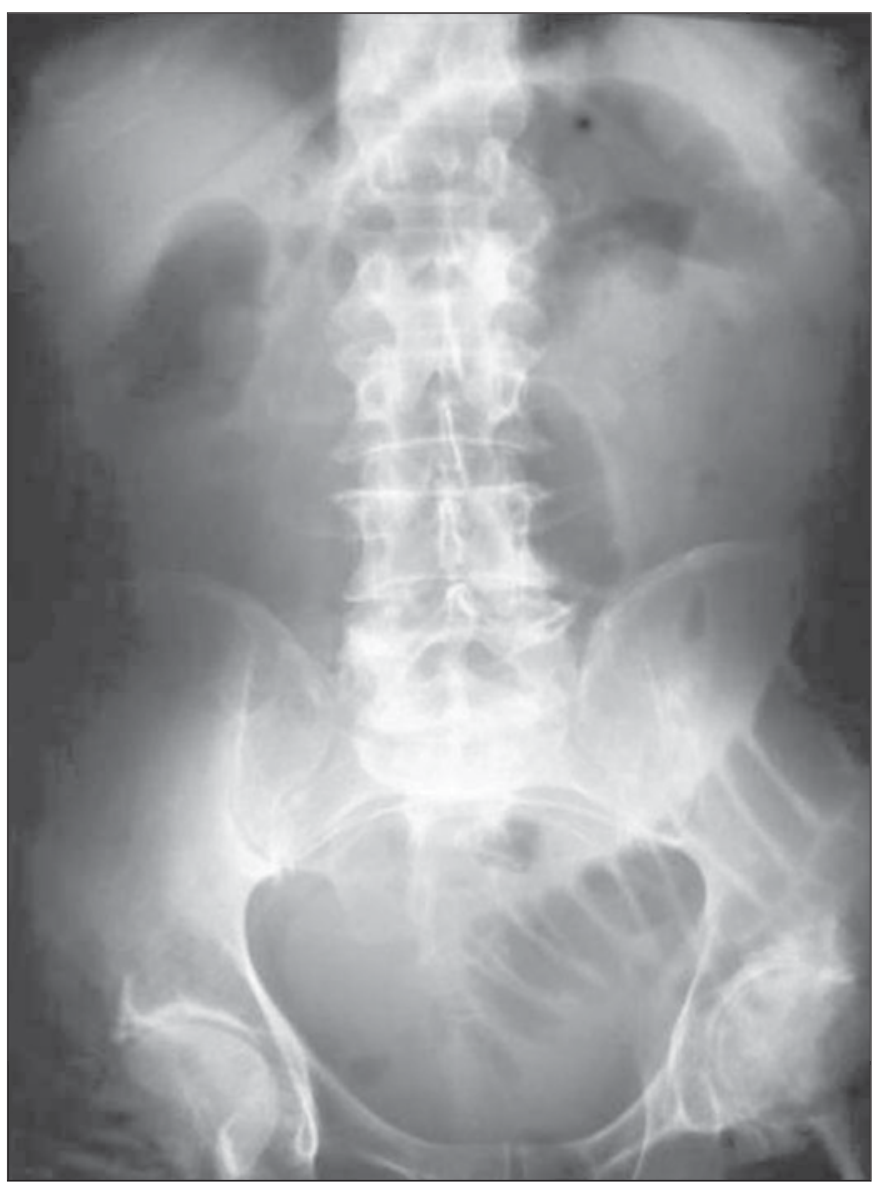

FIGURA 1. Aspecto radiológico de doente com PAF que evoluiu com obstrução intestinal no pós-operatório tardio

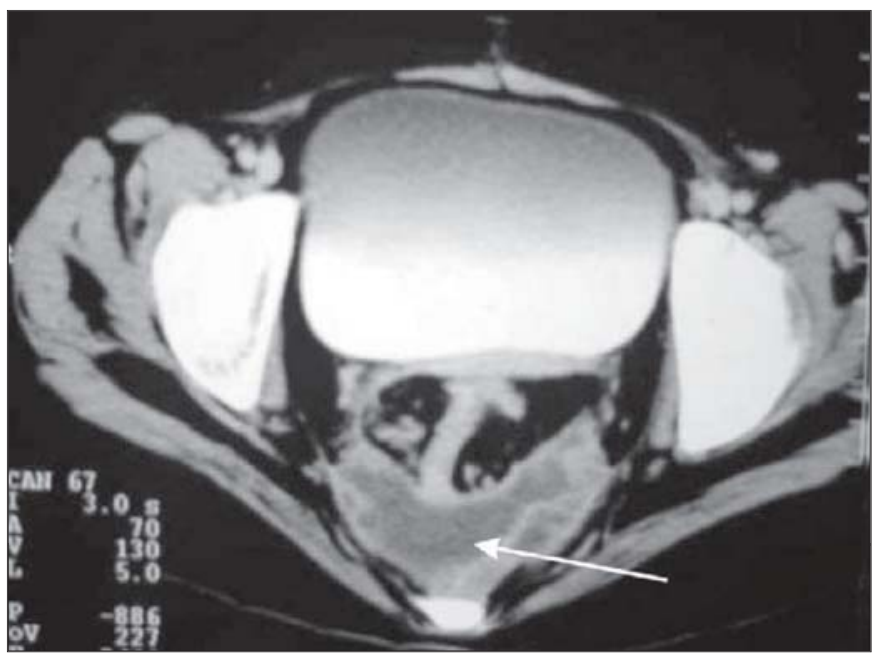

FIGURA 2. Tomografia computadorizada de doente com PAF que evoluiu com abscesso pélvico pré-sacral 
Complicações decorrentes do fechamento da ileostomia ocorreram em quatro casos $(5,8 \%)$, sendo dois abscessos intracavitários, que necessitaram de drenagem cirúrgica. Um doente evoluiu com enterite pseudomembranosa e óbito. Outro apresentou abdome agudo obstrutivo e perfurativo após 3 anos da reconstrução do trânsito intestinal, evidenciando-se à laparotomia, distensão de alças de delgado com perfuração do íleo à montante do local de fechamento da ileostomia, que se apresentava com estenose. Realizou-se sutura da perfuração, dilatação da estenose, lavagem da cavidade e drenagem. O doente evoluiu para óbito no $15^{\circ}$ dia de pós-operatório por choque séptico.

A ileíte do reservatório ou "pouchitis" foi evidenciada através do exame endoscópico em dois doentes $(2,9 \%)$ no pós-operatório tardio. Eles apresentavam febre, aumento do número de evacuações e mialgia. As manifestações clínicas e endoscópicas regrediram após o uso de antibióticos e enemas de corticóide.

Estenose da alça aferente ao RI ocorreu em dois casos (2,9\%), com resolução após dilatação com balão pneumático por via endoscópica.

Fístulas relacionadas ao RI ocorreram em dois doentes $(2,9 \%)$, sendo uma fístula reservatório-vaginal $(1,4 \%)$, tratada com exteriorização do reservatório na parede abdominal, reservatório refeito (re-reservatório) 24 meses após. Em um doente $(1,4 \%)$ ocorreu deiscência parcial da anastomose diagnosticada por meio de exame radiográfico contrastado, antes do fechamento da ileostomia.

Um doente (1,4\%) evoluiu com abscesso abdominal após 15 dias de uma laparotomia por obstrução intestinal devido a aderências, sendo esta no $25^{\circ}$ dia de pós-operatório da cirurgia do reservatório. Realizou-se drenagem cirúrgica com boa evolução (Figura 3).

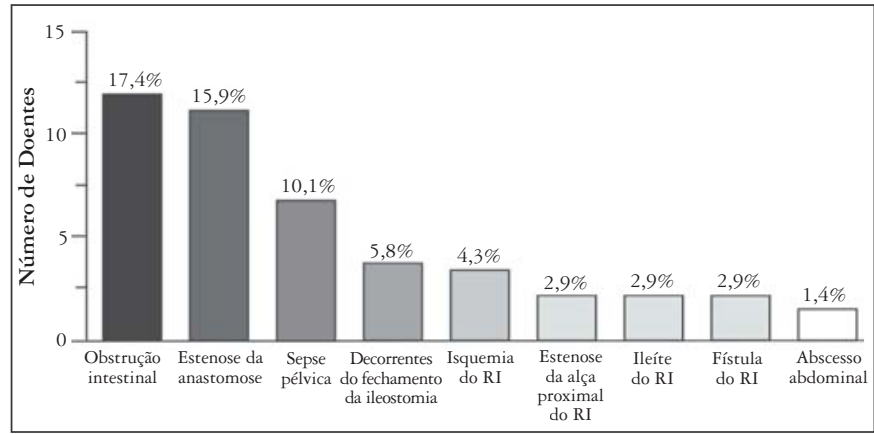

FIGURA 3. Complicações no pós-operatório da cirurgia de reservatório ileal na PAF

Dois óbitos (2,9\%), já mencionados anteriormente, ocorreram no pós-operatório tardio, na evolução de complicações da cirurgia de fechamento da ileostomia. A morbidade, em geral, foi verificada em 44 doentes $(63,8 \%)$.

\section{DISCUSSÃO}

Desde a primeira descrição do emprego do RI por PARKS e NICHOLLS ${ }^{(30)}$, em 1978, é aceito que a retocolectomia total com confecção do RI é a cirurgia ideal para o tratamento da PAF com acometimento colorretal, sendo associada à baixa mortalidade e morbidade aceitável, como visto nesta casuística, sendo de 2,9\% e $63,8 \%$, respectivamente, ressaltando-se que a mortalidade foi devida a complicações decorrentes do fechamento da ileostomia. Estes dados são próximos aos da literatura, que variaram entre $0 \%$ e $0,9 \%$ para mortalidade $(7,8,9,18,21,24,29,34,35)$ e a taxa de morbidade variando de $13 \%$ a $63 \%$. No entanto, em muitos estudos não foram incluídas todas as complicações, sendo enfocadas apenas aquelas consideradas mais importantes ${ }^{(8,26,32,39)}$. No presente estudo considerou-se como morbidade e mortalidade, todas as complicações imediatas, tardias e inclusive as decorrentes do fechamento da ileostomia. Ressalte-se que a colectomia total com anastomose ileorretal constitui a melhor opção para os doentes com PAF com pouco ou nenhum acometimento retal, os quais não foram abordados neste estudo.

LOVEGROVE et al. ${ }^{(24)}$ em uma metanálise, verificaram obstrução intestinal em $17,7 \%$ dos pacientes com PAF operados. Outro estudo ${ }^{(35)}$ mostrou $10 \%$ de obstrução intestinal após o fechamento da ileostomia de proteção, que necessitou de laparotomia. DOZOIS et al. ${ }^{(7)}$ verificaram $19 \%$, sendo que $5 \%$ apenas necessitaram de tratamento cirúrgico. A obstrução intestinal é complicação comum verificada após a cirurgia do RI, sendo de $17,4 \%$ no presente estudo.

A incidência da estenose cicatricial da anastomose ileoanal variou de $5 \%$ a $38 \%$ na literatura ${ }^{(23,26,32,37)}$. Esta grande diferença pode ser explicada em parte, pela variabilidade na definição das estenoses. PRUDHOMME et al. ${ }^{(32)}$ verificaram que $12 \%$ dos doentes com PAF com RI evoluíram para estenose da anastomose, sendo que as estenoses com componente fibroso importante eram devidas, principalmente, a complicações como abscesso, fístulas e retração do RI. Na presente casuística, observaram-se $15,9 \%$ desta complicação, no entanto todas foram resolvidas com dilatação anal, não sendo necessária ressecção da fibrose com avanço mucoso, como é utilizado nas estenoses com fibrose mais acentuada. Desta forma, pode-se verificar que não constituíram morbidade importante para os doentes.

A isquemia aguda do reservatório constitui uma das causas de perda do RI, com necessidade de laparotomia e exérese do mesmo. A isquemia pode ser também lenta e tardia e, neste caso, poderá ocorrer disfuncionalização progressiva com fibrose do RI e até necessidade de remoção do mesmo. LOVEGROVE et al. ${ }^{(24)}$ mencionaram 4,5\% e LEPISTÖ et al. ${ }^{(22)}, 5,3 \%$ de falência do reservatório por isquemia, próximo ao encontrado neste estudo, de 4,3\%. Mesmo assim, em dois dos três casos que evoluíram com isquemia do RI, esta poderia ter sido atribuída a não preservação da arcada do cólon direito, pois foram operados no início da casuística, quando não se realizava esta técnica ${ }^{(13)}$. Ainda, deve-se destacar que as anastomoses sempre foram realizadas na linha pectínea, demandando maior alongamento do mesentério do delgado e, portanto, mais sujeitos à isquemia do RI.

Estenose da alça aferente ao RI, bem como complicações decorrentes do fechamento da ileostomia não foram mencionadas isoladamente na literatura. Apesar desta última complicação ser inerente a qualquer procedimento de fechamento de estoma, nos doentes de PAF com reservatório ileal pode, eventualmente, resultar em maior morbidade. 
A ileíte do reservatório ileal ou "pouchitis" ocorre na PAF em $3 \%$ a $14 \%$ dos casos, sendo uma complicação importante no pós-operatório tardio ${ }^{(1,19,24,25,28,41)}$. A causa desta inflamação inespecífica ainda é desconhecida, mas está associada à presença de crescimento acentuado de bactérias, devido à atrofia da mucosa intestinal secundária ao processo inflamatório ${ }^{(5)}$. O diagnóstico se baseia em aspectos clínicos, endoscópicos e histológicos ${ }^{(36)}$. No entanto, este último aspecto parece não interferir substancialmente para o diagnóstico, como SHEN et al. ${ }^{(38)}$ mostraram por meio do índice de atividade de "pouchitis" modificado. Observou-se na presente casuística $2,9 \%$ de ileíte do reservatório, índice este abaixo do referido pela literatura. Pode-se considerá-lo como sendo baixo e provavelmente deva-se ao fato de se fazer a preservação da arcada do cólon direito, o que impediria isquemias subclínicas, que desencadeariam o processo inflamatório.

A ocorrência de fístula relacionada ao RI no presente estudo (2,9\%) está abaixo da encontrada em outras casuísticas, de $5 \%$ a $16 \%(6,33,40)$. A presença de fístula, que pode ser vaginal, perianal ou perineal, está mais relacionada à doença de base do que à própria técnica cirúrgica utilizada ${ }^{(10)}$, ou seja, os doentes com PAF têm baixo risco para esta complicação, quando comparados aos doentes com doença inflamatória intestinal, submetidos a cirurgia do reservatório ileal ${ }^{(31,33,40)}$. Da mesma maneira, apesar da sepse pélvica estar mais relacionada aos doentes com doença inflamatória intestinal, esta complicação é causa importante de morbidade no pós-operatório da cirurgia do RI na PAF. Observaram-se 10,1\% de evolução com sepse pélvica neste estudo, próximo ao encontrado na literatura. HEUSCHEN et al. ${ }^{(20)}$ observaram $9,2 \%$ em sua casuística e TJANDRA et al. ${ }^{(41)}$, $8 \%$ nos doentes com PAF. No entanto, LOVEGROVE et al. ${ }^{(24)} \mathrm{em}$ metanálise recente, encontraram apenas $1,7 \%$ de ocorrência de sepse pélvica, o que diferiu do resultado encontrado na presente casuística e em todos os demais apresentados.

De qualquer modo, as fístulas e a sepse pélvica são as causas de morbidade após a cirurgia do RI, de mais difícil manejo e que freqüentemente deixam seqüelas ou mesmo resultam na perda do reservatório. Os possíveis fatores que podem desencadear o acúmulo de secreção purulenta na pelve são, principalmente, ocorrência de hematomas retidos e infectados, localizados posteriormente ao RI, e fístulas da sutura do reservatório ileal.

Têm-se observado tendência à diminuição do número de complicações nas análises realizadas no decorrer de outros estudos que revelam a experiência do próprio grupo ${ }^{(11,14,15,16)}$. Assim, a recomendação é que tais procedimentos devam ser realizados por cirurgiões experientes na indicação do tratamento cirúrgico da PAF com escolha da técnica adequada. É importante que tenham experiência na preparação da arcada vascular do íleo terminal visando o RI alcançar com facilidade o canal anal para se evitar tensão na anastomose, assim como comprometimento da viabilidade do RI. Por último, lembrar que o RI excisado por complicações pode ser refeito e que tal procedimento pode ainda oferecer boa qualidade de vida ao doente ${ }^{(14)}$.

\section{CONCLUSÕES}

A morbidade e mortalidade de 63,8\% e 2,9\%, respectivamente, foram de acordo com o observado na literatura, e aceitáveis para uma cirurgia complexa como é a retocolectomia total com confecção do reservatório ileal, levado à linha pectínea e em dois tempos operatórios. Apesar da obstrução intestinal ter sido a complicação mais freqüente, é de fácil resolução na maioria dos casos e mesmo aqueles que necessitaram de laparotomia, a morbidade foi pequena. A isquemia do reservatório ileal e a sepse pélvica foram menos freqüentes, mas constituíram importante complicação relacionada à perda ou disfuncionalização do reservatório.

A crescente experiência com este procedimento tem propiciado maior segurança na sua execução e, apesar de ainda existir percentagem razoável de complicações, estas têm sido menos significativas, o que tem estimulado a sua indicação. Desta forma, é importante atentar para os fatores que eventualmente possam desencadear estas complicações e tentar evitá-las. Além disso, estes resultados têm implicações importantes como informações aos doentes com PAF que são candidatos à cirurgia do reservatório ileal.

Leal RF, Ayrizono MLS, Coy CSR, Fagundes JJ, Góes JRN. Short-term and long-term postoperative complications after ileal pouch-anal anastomosis in familial adenomatous polyposis. Arq Gastroenterol. 2008;45(2):106-10.

ABSTRACT - Background - Restorative proctocolectomy is the procedure of choice to treat familial adenomatous polyposis, however it can be associated to short-term and long-term postoperative complications. Aim - To evaluate the occurrence of complications related to the surgical treatment of familial adenomatous polyposis with ileal pouch technique. Methods - Retrospective study of 69 patients with familial adenomatous polyposis after rectocolectomy with ileal reservoir between 1984 and 2006, operated on Coloproctology Group, Medical Sciences Faculty, State University of Campinas, Campinas, SP, Brazil. The median follow-up period was 82 (2-280) months. Data obtained were surgical techniques and postoperative complications. Results - The morbidity and mortality were $63.8 \%$ and $2.9 \%$, respectively. The most frequent complications were small-bowel obstruction (17.4\%), anastomotic stricture (15.9\%) and pelvic sepsis $(10.1 \%)$. Acute ischemia of the ileal pouch $(4.3 \%)$, pouchitis $(2.9 \%)$ and ileal pouch-related fistula $(2.9 \%)$ had poorer frequency than others. Conclusions - The morbid-mortality was similar to the literature's data and it is acceptable for a complex surgery in two terms like the ileal reservoir-anal anastomosis. The small-bowel obstruction was the most frequent complication. However, ischemia of the reservoir, pouchitis and pelvic sepsis were important complications and was related to the failure of the ileal reservoir.

HEADINGS - Adenomatous polyposis coli, surgery. Ileum, surgery. Rectum, surgery. Colonic pouches. Colectomy, complications 


\section{REFERÊNCIAS}

1. Barton JG, Paden MA, Lane M, Postier RG. Comparison of postoperative outcomes in ulcerative colitis and familial polyposis patients after ileoanal pouch operations. Am J Surg. 2001;182:616-20.

2. Bulow S. Familial polyposis coli. Dan Med Bull. 1987;34:1-15.

3. Bulow S. Incidence of associated diseases in familial polyposis coli. Semin Surg Oncol. 1987;3:84-7.

4. Campos FG. Polipose adenomatosa familiar. Revisão sobre as características clínicas, bases moleculares, tratamento cirúrgico e manuseio das manifestações extra-colônicas. GED Gastroenterol Endosc Dig. 2006;25:37-41.

5. Coffey JC, Bennett MW, Wang JH, O'Connell J, Neary P, Shanahan F, Redmond HP, Kirwan WO. Upregulation of Fas-Fas-L(CD95/CD95L)-mediated epithelial apoptosis - a putative role in pouchitis? J Surg Res. 2001;98:27-32.

6. Dozois RR, Goldberg SM, Rothenberger DA, Utsunomiya J, Nicholls RJ, Cohen Z Hulten LA, Moskowitz RL, Williams NS. Restorative proctocolectomy with ileal reservoir. Int J Colorectal Dis. 1986;1:2-19.

7. Dozois RR, Kelly KA, Welling DR, Gordon H, Beart RW, Wolff BG, Pemberton JH, Ilstrup DM. Ileal pouch-anal anastomosis: comparison of results in familia adenomatous polyposis and chronic ulcerative colitis. Ann Surg. 1989;210:268-73.

8. Fazio VW, Tekkis PP, Remzi F, Lavery IC, Manilich E, Connor J, Preen M, Delaney CP. Quantification of risk for pouch failure after ileal pouch anal anastomosis surgery. Ann Surg. 2003;238:605-17.

9. Fazio VW, Ziv Y, Church JM, Oakley JR, Lavery IC, Milsom JW, Schroeder TK. Ilea pouch-anal anastomoses complications and function in 1005 patients. Ann Surg. 1995;222:120-7.

10. Gecim IE, Wolff BG, Pemberton JH, Devine RM, Dozois RR. Does technique of anastomosis play any role in developing late perianal abscess or fistula? Dis Colon Rectum. 2000;43:1241-5.

11. Góes JRN, Fagundes JJ, Costa AM, Peres MAO, Medeiros RR, Leonardi LS. Reservatório ileal pélvico. Detalhes de técnica operatória e avaliação das complicações. Rev Bras Coloproctol. 1987;7:94-8.

12. Góes JRN, Fagundes JJ, Coy CSR, Amaral CAR, Peres MAO, Medeiros RR. The two-chamber ileal pelvic reservoir - an alternative design. Dis Colon Rectum. 1993;36:403-4.

13. Góes JRN, Fagundes JJ, Coy CSR, Amaral CAR, Oliveira C, Ayrizono MLS, Medeiro RR. O emprego da artéria cólica média e da arcada vascular do cólon direito na irrigação de reservatórios ileais. Rev Bras Coloproctol. 1994;14:169-71.

14. Góes JRN, Coy CSR, Ayrizono MLS, Fagundes JJ, Medeiros RR. Re-reservatório ilea pélvico. Reconstrução e reanastomose de reservatório ileal pélvico após insucesso da primeira cirurgia. Rev Bras Coloproctol. 1997;17:117-21.

15. Góes JRN, Fagundes JJ, Coy CSR, Ayrizono MLS, Medeiros RR, Leonardi LS Retocolectomia total e anastomose íleo-anal com reservatório ileal: experiência de 16 anos. Rev Bras Coloproctol. 2000;27:33-9.

16. Góes JRN, Fagundes JJ, Coy CSR, Ayrizono MLS, Moreira APP, Leonardi LS Retocolectomia total, anastomose íleo-anal com reservatório ileal e ocorrência de obstrução intestinal. Rev Bras Coloproctol. 2004;24:329-33.

17. Guillen JG, Wood WC, Moley JF, Berchuck A, Karlan BY, Mutch DG, Gagel RF, Weitzel J, Morrow M, Weber BL, Giardiello F, Rodriguez-Bigas MA, Church J, Gruber $\mathrm{S}$, Offit K. ASCO/SSO Review of current role of risk-reducing surgery in common hereditary cancer syndromes. Ann Surg Oncol. 2006;13:1296-321.

18. Heuschen UA, Autschbach F, Allemeyer EH, Zöllinger AM, Heuschen G, Uehlein T, Herfarth C, Stern J. Long-term follow-up after ileoanal pouch procedure. Algorithm for diagnosis, classification, and management of pouchitis. Dis Colon Rectum. 2001;44:487-99.

19. Heuschen UA, Heuschen G, Lucas M, Hinz U, Stern J, Herfarth C. Prä-und postoperative lebensqualität von patienten mit colitis ulcerosa und familiärer adenomatöser polyposis bei ileoanaler pouchoperation. Chirurg. 1998;69:1329-33.

20. Heuschen UA, Hinz U, Allemeyer EH, Austschbach F, Stern J, Lucas M, Herfarth C, Heuschen G. Risk factors for ileoanal J pouch-related septic complications in ulcerative colitis and familial adenomatous polyposis. Ann Surg. 2002;235:207-16.
21. Körsgen S, Keighley MRB. Causes of failure and life expectancy of the ileoanal pouch. Int J Colorectal Dis. 1997;12:4-8.

22. Lepistö A, Luukkonen P, Järvinen HJ. Cumulative failure rate of ileal pouch-ana anastomosis and quality of life after failure. Dis Colon Rectum. 2002;45:1289-94.

23. Lewis WG, Kuzu A, Sagar PM, Holdsworth PJ, Johnston D. Stricture at the pouch-anal anastomosis after restorative proctocolectomy. Dis Colon Rectum. 1994:35:120-5.

24. Lovegrove E, Tilney HS, Heriot AG, von Roon AC, Athanasiou T, Church J, Fazio VW, Tekkis PP. A comparison of adverse events and functional outcomes after restorative proctocolectomy for familial adenomatous polyposis and ulcerative colitis. Dis Colon Rectum. 2006;49:1293-306.

25. Macafee DAL, Abercrombie JF, Armstrong CM. Pouchitis. Colorectal Dis. 2004;6:142-52.

26. Marcello PW, Roberts PL, Schoëtz DJJr, Coller JA, Murray JJ, Veidenheimer MC Long-term results of the ileo-anal pouch procedure. Arch Surg. 1993;128:500-3.

27. Nicholls RJ. Restorative proctocolectomy with various types of reservoir. World J Surg. 1987;11:751-62.

28. Nicholls RJ, Banerjee AK. Pouchitis: risk factors, etiology, and treatment. World J Surg. 1998;22:347-56.

29. Parc Y, Piquard A, Dozois RR, Parc R, Tiret E. Long-term outcome of familial adenomatous polyposis patients after restorative coloproctectomy. Ann Surg. 2004;239:378-82.

30. Parks AG, Nicholls RJ. Proctocolectomy without ileostomy for ulcerative colitis. $\mathrm{Br}$ Med J. 1978;2:85-8.

31. Paye F, Penna C, Chiche L, Tiret E, Frileux P, Parc R. Pouch-related fistula following restorative proctocolectomy. Br J Surg. 1996;83:1574-7.

32. Prudhomme M, Dozois RR, Godlewski G, Mathison S, Fabbro-Peray P. Anal canal strictures after ileal pouch-anal anastomosis. Dis Colon Rectum. 2003;46:20-3.

33. Richard CS, Cohen Z, Stern HS, McLeod RS. Outcome of the pelvic pouch procedure in patients with prior perianal disease. Dis Colon Rectum. 1997;40:647-52.

34. Romanos J, Samarasekera DN, Stebbing JF, Jewell DP, Kettlewell MGW, Mortensen NJM. Outcome of 200 restorative proctocolectomy operations: the John Radcliffe Hospital experience. Br J Surg. 1997;84:814-8.

35. Salemans JMJI, Nagengast FM, Lubbers EJC, Kuijpers JH. Postoperative and longterm results of ileal pouch-anal anastomosis for ulcerative colitis and familial polyposis coli. Dig Dis Sci. 1992;37:1882-9.

36. Sandborn WJ, Tremaine WJ, Batts KP, Pemberton JH, Phillips SF. Pouchitis after ileal pouch-anal anastomosis: a pouchitis disease activity index. Mayo Clin Proc. 1994;69:409-15.

37. Senapati A, Tibbs CJ, Ritchie JK, Nicholls RJ, Hawley PR. Stenosis of the pouch anal anastomosis following restorative proctocolectomy. Int J Colorectal Dis. 1996;11:57-9.

38. Shen B, Achkar JP, Connor JT, Ormsby AH, Remzi FH, Bevins CL, Brzezinski A, Bambrick ML, Fazio VW, Lashner BA. Modified pouchitis disease activity index. A simplified approach to the diagnosis of pouchitis. Dis Colon Rectum. 2003;46:748-53.

39. Skarsgard ED, Arkinson KG, Bell GA, Pezim ME, Seal AM, Sharp FR. Function and quality of life results after ileal pouch surgery for chronic ulcerative colitis and familial polyposis. Am J Surg. 1989;157:467-71.

40. Tekkis PP, Fazio VW, Remzi F, Heriot AG, Manilich E, Strong SA. Risk factors associated with ileal pouch-related fistula following restorative proctocolectomy. $\mathrm{Br}$ J Surg. 2005;92:1270-6.

41. Tjandra JJ, Fazio VW, Church JM, Oakley JR, Milson JW, Lavery IC. Similar functional results after restorative proctocolectomy in patients with familial adenomatous polyposis and mucosal ulcerative colitis. Am J Surg. 1993;165:322-5.

42. Utsunomiya J, Iwama T, Imajo M, Matsuo S, Sawai S, Yaegashi k, Hirayama R. Tota colectomy, mucosal proctectomy, and ileoanal anastomosis. Dis Colon Rectum. 1980;23:459-66 